\title{
Velocity estimation using recursive ultrasound imaging and spatially encoded signals
}

\author{
Nikolov, Svetoslav; Gammelmark, Kim; Jensen, Jørgen Arendt
}

\section{Published in:}

Ultrasonics Symposium, 2000 IEEE

Link to article, DOI:

10.1109/ULTSYM.2000.921602

Publication date:

2000

Document Version

Publisher's PDF, also known as Version of record

Link back to DTU Orbit

Citation (APA):

Nikolov, S., Gammelmark, K., \& Jensen, J. A. (2000). Velocity estimation using recursive ultrasound imaging and spatially encoded signals. In Ultrasonics Symposium, 2000 IEEE (pp. 1473-1477). IEEE.

https://doi.org/10.1109/ULTSYM.2000.921602

\section{General rights}

Copyright and moral rights for the publications made accessible in the public portal are retained by the authors and/or other copyright owners and it is a condition of accessing publications that users recognise and abide by the legal requirements associated with these rights.

- Users may download and print one copy of any publication from the public portal for the purpose of private study or research.

- You may not further distribute the material or use it for any profit-making activity or commercial gain

- You may freely distribute the URL identifying the publication in the public portal 


\title{
Velocity estimation using recursive ultrasound imaging and spatially encoded signals
}

\author{
Svetoslav Nikolov, Kim Gammelmark and Jørgen Jensen \\ Center for Fast Ultrasound Imaging, Department of Information Technology, Build. 344, \\ Technical University of Denmark, DK-2800 Lyngby, Denmark
}

\begin{abstract}
Previously we have presented a recursive beamforming algorithm for synthetic transmit aperture focusing. At every emission a beamformed low-resolution image is added to an existing high-resolution one, and the low-resolution image from the previous emission with the current active element is subtracted yielding a new frame at every pulse emission.

In this paper the method is extended to blood velocity estimation, where a new Color Flow Mapping (CFM) image is created after every pulse emission. The underlying assumption is that the velocity is constant between two pulse emissions and the current estimates can therefore be used for compensation of the motion artifacts in the data acquired in the next emission.

Two different transmit strategies are investigated in this paper: (a) using a single defocused active aperture in transmit, and (b) emitting with all active transmit sub-apertures at the same time using orthogonal spatial encoding signals.

The method was applied on data recorded by an experimental system. The estimates of the blood velocity for both methods had a bias less than $3 \%$ and a standard deviation around $2 \%$ making them a feasible approach for blood velocity estimations.
\end{abstract}

\section{Introduction}

Modern scanners estimate the blood velocity by sending ultrasound pulses in the same direction and processing the signal returned from a given depth. To create a map of the velocity distribution in the area of investigation, the signal must be sent several consecutive times in each of several different directions. The precision of the estimates increases, if the estimates are based on a larger number of acquisitions in one direction. This, however, decreases the frame rate and the choice is based on a compromise between frame rate and precision.

This compromise can be avoided if a new frame is created after every emission and its data used for velocity estimation.
The continuous flow of data allows the use of stationary echo canceling filters with longer impulse responses, and estimates based on a larger number of emissions, which both improve the estimates' precision.

One approach to create a new frame at every pulse emission is to use Recursive Ultrasound Imaging [1]. The beamformed data as proposed in [1] is suitable for B-mode imaging but not for blood velocity estimation, because of the present motion artifacts.

In this article the CFM is calculated after every emission, and the velocity estimates from the current frame are used for correcting the motion artifacts in the next one. Since the estimates are based on longer sample sequences, they have a high precision, and the motion artifacts can thereby be compensated fully.

Since each emission is performed only by one element, and the blood is moving, the performance of the above mentioned procedure depends on the shot sequence. This dependency can be avoided by using the same elements at every emission with a spatial encoding scheme as suggested in [2].

\section{Theoretical background}

The following sections give the theoretical background for recursive ultrasound imaging and the use of spatially encoded transmits to increase the signal-to-noise ratio.

\subsection{Recursive imaging}

A pulse emitted by only one transducer element propagates as a spherical wave, when the element is small, and the received echo signal carries information from the whole region of interest. By applying different delays in receive, any of the scan-lines $m \in\left[1 \ldots N_{m}\right]$ can be formed. The data from one emission is used to beam-form all of the scan-lines creating one image as shown in Fig. 1. The created image has a low resolution, since only one element is used for emission. A high-resolution image is created by summing the RF lines from $N_{x m t}$ low resolution images, each of them created after 


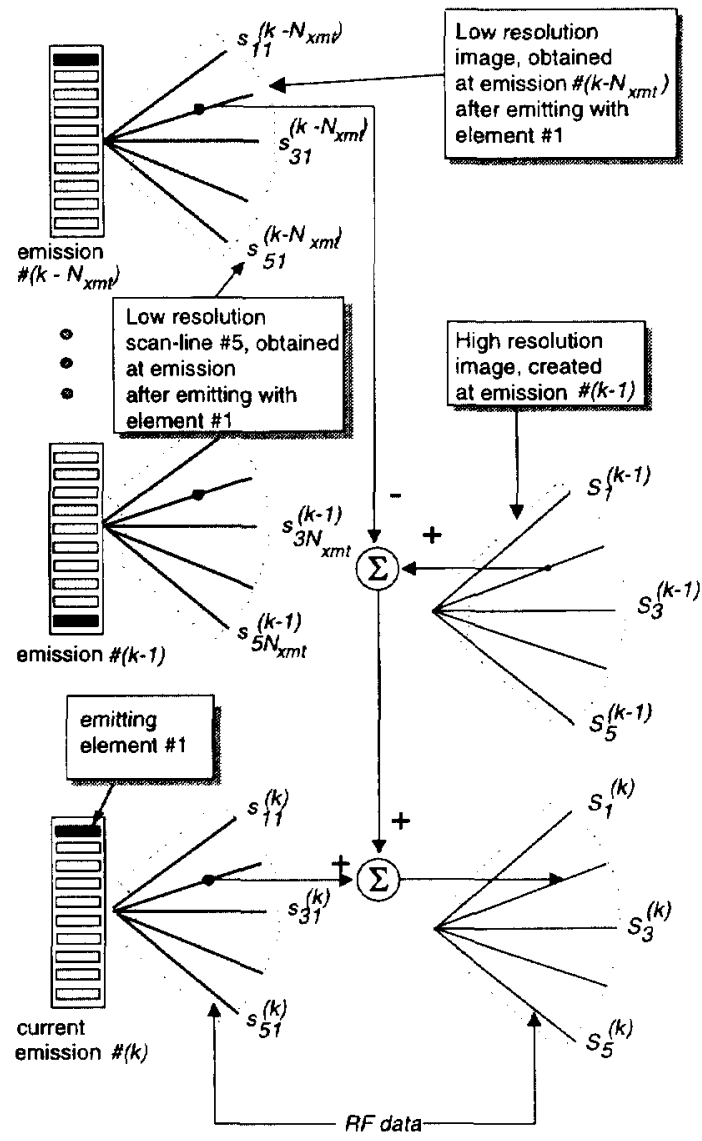

Figure 1: Recursive ultrasound imaging. In transmit only one element is excited. Multiple receive beams are formed simultaneously for each transmit pulse. Each element is excited again after $N_{x m t}$ emissions $\left(N_{x m t}=N_{x d c}=10\right.$ in this example).

emitting with a different transducer element. Let the number of the current emission be $k$, the number of the transducer elements be $N_{x d c}$, the recorded signal by the element $j$ after emitting with element $i$ be $r_{i j}^{(k)}$, and let the necessary delay and the weighting coefficient for beam-forming of scan-line $m$ be $d_{m i j}$ and $a_{m i j}$, respectively. The beam-forming of a scanline for a low-resolution image can then be expressed as (see Fig. 1):

$$
s_{m i}^{(k)}(t)=\sum_{j=1}^{N_{x d c}} a_{m i j} \cdot r_{i j}^{(k)}\left(t-d_{m i j}\right)
$$

where $t$ is time relative to start of pulse emission. Provided that the tissue below the transducer is motionless, the forming of the final scan-lines for the high-resolution image can be expressed as [1]:

$$
S_{m}^{(k)}(t)=S_{m}^{(k-1)}(t)+s_{m i}^{(k)}(t)-s_{m i}^{\left(m-N_{x m t}\right)}(t)
$$

This method, however, suffers from a low signal-to-noise (SNR) ratio and from motion" artifacts. Using multiple elements in transmit to send "defocused" ultrasound wave improves [3] the situation. Further, the SNR can be increased by using encoded signals. The encoding can be temporal (for example using linear frequency modulated excitation) or spatial as described in the next section.

\subsection{Spatial encoding}

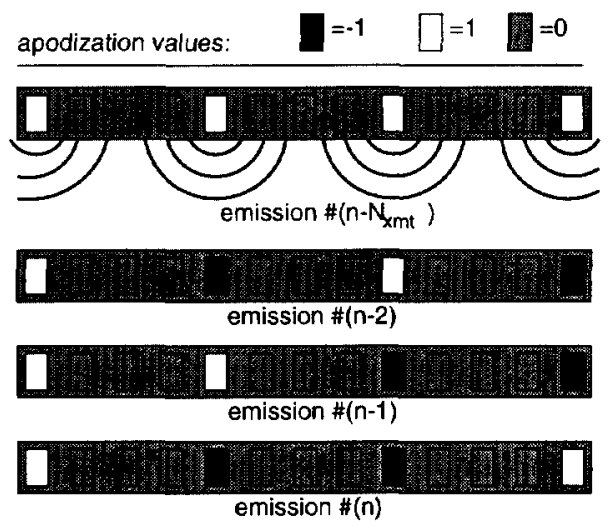

Figure 2: Spatially encoded transmits using 4 transmit elements.

The idea behind the spatial encoding is to send with all of the $N_{x m t}$ elements as shown in Fig. 2, instead of sending with only one element $i, 1 \leq i \leq N_{x m t}$ at a time [2]. The signal sent into the tissue by each of the transmit elements $i$ is

$$
e_{i}(t)=q_{i} \cdot e(t), \quad 1 \leq i \leq N_{x m t},
$$

where $e(t)$ is a basic waveform and $q_{i}$ is an encoding coefficient.

Assuming a linear propagation medium, the signal $r_{j}(t)$ received by the $j$ th element can be expressed as:

$$
r_{j}(t)=\sum_{i=1}^{N_{x m t}} q_{i} \cdot r_{i j}(t)
$$

where $r_{i j}(t)$ would be the signal received by element $j$, if the emission was done only by element $i$.

From Eq.(1) it can be seen that the components $r_{i j}(t)$ must be found in order to beamform the signal. The received signals can be expressed in a matrix form:

$$
\left[\begin{array}{c}
r_{j}^{(1)} \\
r_{j}^{(2)} \\
\vdots \\
r_{j}^{\left(N_{x m t}\right)}
\end{array}\right]=\left[\begin{array}{cccc}
q_{1}^{(1)} & q_{2}^{(1)} & \cdots & q_{N_{x m t}}^{(1)} \\
q_{1}^{(2)} & q_{2}^{(2)} & \cdots & q_{N_{x m t}}^{(2)} \\
\vdots & \vdots & \ddots & \vdots \\
q_{1}^{\left(N_{x m t}\right)} & q_{2}^{\left(N_{x m t}\right)} & \cdots & q_{N_{x m t}}^{\left(N_{x m t}\right)}
\end{array}\right]\left[\begin{array}{c}
r_{1 j} \\
r_{2 j} \\
\vdots \\
r_{N_{x m t} j}
\end{array}\right]
$$


where the superscript ${ }^{(k)}, 1 \leq k \leq N_{x m t}$ is the number of the emission, $q_{i}^{(k)}$ is the encoding coefficient applied in transmit on the transmitting element $i$, and $r_{j}^{(k)}$ is the signal received by the $j$ th element. In the above system of equations the time is skipped for notational simplicity. Also stationary tissue is assumed so that:

$$
r_{i j}^{(1)}=r_{i j}^{(2)}=\cdots=r_{i j}^{\left(N_{x m t}\right)}=r_{i j}
$$

More compactly, the equation can be written as:

$$
\vec{r}_{j}=\mathbf{Q} \vec{r}_{i j}
$$

where $\mathbf{Q}$ is the encoding matrix. Obviously the responses $r_{i j}(t)$ are:

$$
\overrightarrow{r_{i j}}=\mathbf{Q}^{-1} \vec{r}_{j}
$$

A suitable encoding matrix $\mathbf{Q}$ is the Hadamard matrix $\mathbf{H}$ [2]. The inverse Hadamard matrix is a scaled version of itself, i.e. for a matrix $\mathbf{H}_{N_{x m t}}$ with $N_{x m t} \times N_{x m t}$ elements, the inverse is $\mathbf{H}_{N_{x m t}}^{-1}=1 / N_{x m t} \mathbf{H}_{N_{x m s}}$.

The above derivation strongly relies on the assumption in (6). In the case of abdominal scanning, and for low values of $N_{x m t}$, this assumption is "almost fulfilled". However, in cardiac imaging and blood velocity estimation, this assumption is severely violated and the movement of the blood and heart must be compensated for.

\subsection{Motion compensation}

The motion compensation is considered for two cases: (a) recursive imaging without spatial encoding and (b) recursive imaging with spatial encoding.

\subsubsection{Without spatial encoding}

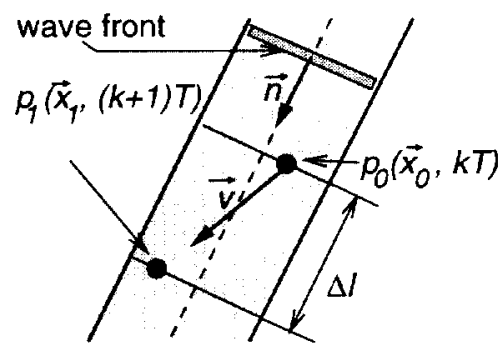

Figure 3: Motion compensation for recursive imaging without spatial encoding.

During the first stage of the beamforming process, lowresolution images are created, using dynamic receive focusing. The assumption is that within one scan line $s_{m i}(t)$, the wavefront propagates as a plane wave, as shown in Fig. 3.
Figure 3 shows the movement of one point scatterer within the limits of one scan line. The scatterer moves with velocity $\vec{v}$, from position $p_{0}\left(\overrightarrow{x_{0}}, k T\right)$ to a new position $p_{1}\left(\overrightarrow{x_{1}},(k+1) T\right)$ for the time $T$ between two pulse emissions. The movement across the beam (perpendicular to the the direction $\vec{n}$ ) determines the strength of the backscattered energy, while the movement along the beam determines the time instance when the backscattering occurs.

For the case depicted in Fig. 3 the difference in time when the backscattering occurs for the positions $p_{0}$ and $p_{1}$ is:

$$
\tau=\frac{2 \cdot \Delta l}{c}
$$

where $\Delta l$ is the distance traveled from one pulse emission to the next:

$$
\Delta l=\langle\vec{v}, \vec{n}\rangle T
$$

where $\langle\vec{v}, \vec{n}\rangle$ is the inner product between the velocity vector $\vec{v}$ and the directional vector $\vec{n}$.

The velocity at emission $k$ as a function of time $t$ from the emission of the pulse along the line $m$ is $v_{m}^{(k)}(t)$. The delay $\tau$ is also a function of $t, \tau^{(k)}=\tau_{m}^{(k)}(t)$. The beamformation process with the velocity incorporated in it becomes:

$$
\begin{aligned}
& \text { for } m=1 \text { to } N_{m} \\
& \qquad \begin{array}{l}
s_{m i}^{(k)}(t)=\sum_{j=1}^{N_{x d c}} a_{m i j} \cdot r_{i j}^{(k)}\left(t-d_{m i j}\right) \\
M_{m}^{(k)}(t)=S_{m}^{(k-1)}(t)-s_{m i}^{\left(k-N_{x m t}\right)}(t) \\
\tau_{m}^{(k+1)}(t)=\frac{2\left|\vec{v}_{m}^{(k)}(t)\right| T}{c} \\
\Delta_{m}^{(k)}(t)=\Delta_{m}^{(k-1)}(t)+\tau_{m}^{(k)}-\tau_{m}^{\left(k-N_{x m t}+1\right)}(t) \\
S_{m}^{(k)}(t)=M_{m}^{(k)}\left[t+\tau_{m}^{\left(k-N_{x m t}+1\right)}(t)\right]+s_{m i}^{(k)}\left[t-\Delta_{m}^{(k)}(t)\right]
\end{array}
\end{aligned}
$$

where $\Delta$ is the delay between the first and the last of the lowresolution images, currently comprised in the high-resolution one.

\subsubsection{With spatial encoding}

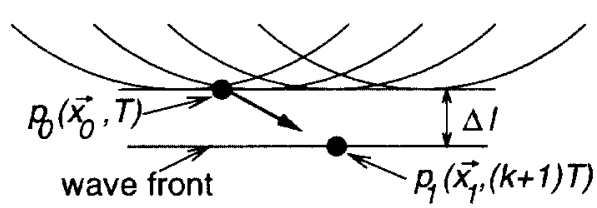

Figure 4: Motion compensation for recursive imaging with spatial encoding.

Figure 4 shows the model adopted for motion compensation in the presence of spatially encoded signals. Several 
transducer elements across the whole span of the transducer aperture are used in transmit. The sum of the emitted waves creates a planar wave propagating in a direction perpendicular to the transducer surface. A point scatterer moves for one pulse-repetition period from positions $p_{0}\left(\vec{x}_{0}, k T\right)$ to a new position $p_{1}\left(\vec{x}_{1},(k+1) T\right)$. The difference between the time instances, when the scattering occurs, is :

$$
\tau=\frac{2 \Delta l}{c},
$$

where $\Delta l$ is the distance traveled by the point scatterer:

$$
\Delta l=v_{z} T
$$

In the above equation $v_{z}$ is the component of the velocity normal to the transducer surface. The delay $\tau$ is a function of the time $t$, and the emission number $k, \tau=\tau^{(k)}(t)$

Thus, the signals received by element $j$ for emission number $k \in\left[1, N_{x m t}\right]$ are:

$$
\begin{aligned}
r_{j}^{(2)}(t) & =r_{j}^{(1)}\left(t-\tau^{(1)}(t)\right) \\
r_{j}^{(3)}(t) & =r_{j}^{(2)}\left(t-\tau^{(2)}(t)\right) \\
\vdots & \\
r_{j}^{\left(N_{x m t}\right)}(t) & =r_{j}^{\left(N_{x m t}-1\right)}\left(t-\tau^{\left(N_{x m t}-1\right)}(t)\right)
\end{aligned}
$$

The reconstruction must be performed prior to beamforming the signal at a given point. First the received signals $r^{(k)}(t)$ are appropriately delayed, and then the system of equations (5) is solved.

\section{Experimental results}

\subsection{Measurement setup}

The measurements were done, using the department's off-line experimental system XTRA [4]. The most important parameters are listed in Table 1.

\begin{tabular}{lccc}
\hline \hline Parameter name & Notation & Value & Unit \\
\hline Speed of sound & $c$ & 1540 & $\mathrm{~m} / \mathrm{s}$ \\
Sampling freq. & $f_{s}$ & 40 & $\mathrm{MHz}$ \\
Excitation freq. & $f_{0}$ & 5 & $\mathrm{MHz}$ \\
Pulse duration & $T_{p}$ & 1.5 & cycles \\
-6 dB band-width & $B W$ & $4.875-10.125$ & $\mathrm{MHz}$ \\
Transducer pitch & $p$ & 209 & $\mu \mathrm{m}$ \\
Transducer kerf & $k e r f$ & 30 & $\mu \mathrm{m}$ \\
Number of elements & $N_{x d c}$ & 64 & - \\
Transducer height & $h$ & 4 & $\mathrm{~mm}$ \\
Elevation focus & $f_{e z}$ & 20 & $\mathrm{~mm}$ \\
\hline \hline
\end{tabular}

Table 1: Measurement parameters
A tissue mimicking phantom with frequency dependent attenuation of $0.25 \mathrm{~dB} /[\mathrm{cm} . \mathrm{MHz}]$ and speed of sound $c=1540$ $\mathrm{m} / \mathrm{s}$ was scanned at 65 positions in a water bath. From position to position the phantom was moved $70 \mu \mathrm{m}$ at an angle of $45^{\circ}$ to the transducer surface. Assuming a pulse repetition frequency $f_{p r f}=1 / T=7000$, this movement corresponds to a plug-flow with velocity $|\vec{v}|=49.5 \mathrm{~cm} / \mathrm{s}$.

A precision translation system was used for the movement of the phantom. The precision of the motuon in the axial and lateral directions were: $\Delta z=1 / 200 \mathrm{~mm}$, and $\Delta x=1 / 80 \mathrm{~mm}$, respectively.

\subsection{Velocity estimation}

In the theoretical considerations, it was assumed that the blood velocity was estimated, without any considerations about the velocity estimator.

The cross-correlation estimator suggested in [5] is suitable for the broad band pulses used by this method. In the implementation it is assumed, that the two consecutive highresolution lines $S^{(k)}\left(t_{2}\right)$ and $S^{(k-1)}\left(t_{1}\right)$ are related by:

$$
S^{(k)}\left(t_{2}\right)=S^{(k-1)}\left(t_{1}-t_{s}\right),
$$

where $t_{s}$ is a time lag due to the movement of the scatterers and is related to the axial component of the velocity $v_{z}$ by:

$$
t_{s}=\frac{2 v_{z}}{c} T
$$

The peak of the cross-correlation between segments of $S^{(k)}(t)$ and $S^{(k-1)}(t)$ would be found at time $\hat{t}_{s}$. Estimating $\hat{t}_{s}$ leads to the estimation of $\vec{v}$.

\subsection{Reference velocity estimation}

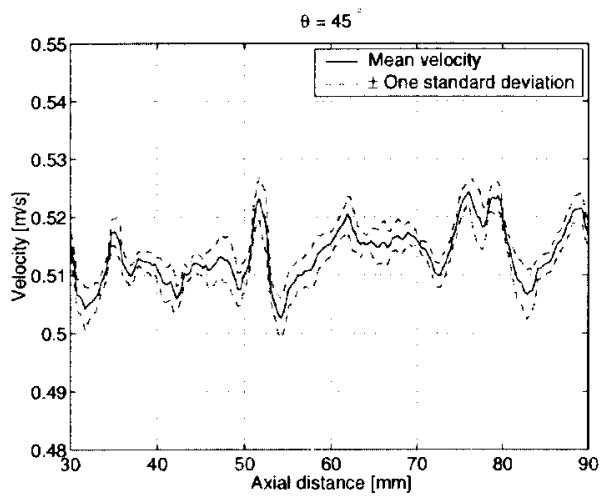

Figure 5: Mean reference velocity.

In order to obtain a reference estimate of the velocity, at each position of the phantom a high resolution image was 


\begin{tabular}{cccc}
\hline \hline & Reference & Spatially encoded & Non encoded \\
\hline $\overrightarrow{\mid v} \mid[\mathrm{m} / \mathrm{s}]$ & 0.496 & 0.486 & 0.479 \\
$\sigma /|\vec{v}| \%$ & 2.3 & 2.2 & 1.8 \\
\hline \hline
\end{tabular}

Table 2: Results from the velocity estimation at angle $\angle(\vec{v}, \vec{n})=45^{\circ}$

created using 13 emissions per image. The velocity was estimated using a cross-correlation estimator. The correlation length was equal to the length of the transmitted pulse. The number of lines, over which the calculated correlation function was averaged was 8 . The search length was $\pm \lambda / 4$ to avoid aliasing problems. Figure 5 shows the mean velocity $\overrightarrow{\mid \vec{v}} \mid$ for the central line as a function of depth. The mean was calculated over 55 estimates.

In the axial direction the translation system has a precision of $\Delta z=5 \mu \mathrm{m}$, which is $10 \%$ of the desired step. The results are, thus, within the precision of the system.

\subsection{Recursive velocity estimation}

The mean velocity $\overline{|\vec{v}|}$ and the normalized standard deviation $\sigma /|\vec{v}|$ estimated using recursive ultrasound imaging are shown in Table 2 . The angle between the velocity vector $\vec{v}$ and the directional vector $\vec{n}$ of the scan line is $\angle(\vec{v}, \vec{n})=45^{\circ}$. The number of frames is 36 . In this table $\overline{|\vec{v}|}$ is the average of the mean velocity in the range from 30 to $80 \mathrm{~mm}$. $\sigma$ is also averaged in the same range. The angle dependence of the estimates is shown in Figure 6. The dashed lines show the velocity at $\pm \sigma$.
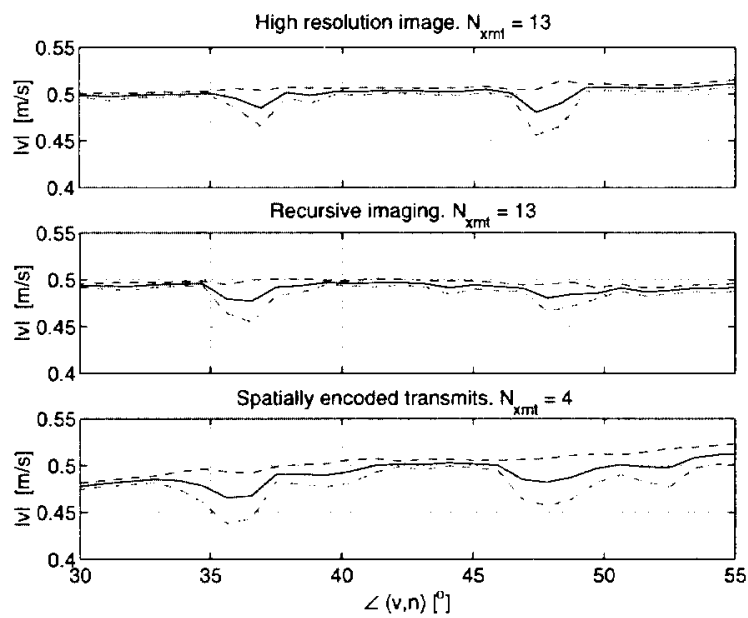

Figure 6: The mean velocity and the velocity at $\pm \sigma$ as a function of angle.

It can be seen that the reference velocity estimation exhibits a smaller bias than the velocity estimations using recursive imaging.
The recursive imaging using spatially encoded transmits exhibits angular dependence. At angles of $42^{\circ}-45^{\circ}$ it has a low bias and standard deviation, comparable to that of the reference velocity estimates. One of the possible reasons for the angular dependency is the low number of emissions $\left(N_{x m t}=4\right)$, resulting in higher side and grating lobes in the image.

\section{Conclusions}

In this paper a method for motion compensation and velocity estimation using recursive ultrasound imaging was presented. The method provides the blood velocity estimator with as much as several thousand measurements per second for every sample in the investigated region.

It has been experimentally verified that the method works for a speckle generating phantom with frequency dependent attenuation. One limitation is that no noise was present in the experiment and the velocity was constant.

Future work will include velocity profiles and mixture of moving and stationary tissue.

\section{Acknowledgements}

This work was supported by grant 9700883 and 9700563 from the Danish Science Foundation and by B-K Medical $\mathrm{A} / \mathrm{S}$.

The measurement system XTRA was built by Søren Kragh Jespersen, as part of his Ph.D. study.

\section{References}

[1] S. I. Nikolov, K. Gammelmark, and J. A. Jensen. Recursive ultrasound imaging. In Proc. IEEE Ultrason. Symp., pages 1621-1625, 1999.

[2] R. Y. Chiao, L. J. Thomas, and S. D. Silverstein. Sparse array imaging with spatially-encoded transmits. In Proc. IEEE Ultrason. Symp., pages 1679-1682, 1997.

[3] G. R. Lockwood, J. R. Talman, and S. S. Brunke. Realtime 3-D ultrasound imaging using sparse synthetic aperture beamforming. IEEE Trans. Ultrason., Ferroelec., Freq. Contr., 45:980-987, 1998.

[4] S. K. Jespersen, J. E. Wilhjelm, and H. Sillesen. Multiangle compound imaging. Ultrason. Imaging, 20:81$102,1998$.

[5] O. Bonnefous and P. Pesqué. Time domain formulation of pulse-Doppler ultrasound and blood velocity estimation by cross correlation. Ultrason. Imaging, 8:73-85, 1986. 